Article

\title{
Improvement of Porous GaN-Based UV Photodetector with Graphene Cladding
}

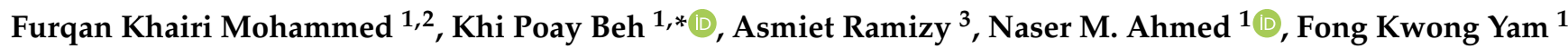 \\ and Zainuriah Hassan ${ }^{4}$ \\ 1 School of Physics, Universiti Sains Malaysia, Minden 11800, Penang, Malaysia; \\ furqan.khairi@student.usm.my (F.K.M.); naser@usm.my (N.M.A.); yamfk@usm.my (F.K.Y.) \\ 2 Department of Radiology and Sonography Techniques, Al-Rafidain University College, Baghdad 10014, Iraq \\ 3 Physics Department, College of Science, University of Anbar, 55 Ramadi, Baghdad 55431, Iraq; \\ asmat_hadithi@uoanbar.edu.iq \\ 4 Institute of Nano Optoelectronics Research and Technology (INOR), Universiti Sains Malaysia, \\ Minden 11800, Penang, Malaysia; zai@usm.my \\ * Correspondence: behkhipoay@usm.my
}

Citation: Mohammed, F.K.; Beh, K.P.; Ramizy, A.; Ahmed, N.M.; Yam, F.K.; Hassan, Z. Improvement of Porous GaN-Based UV Photodetector with Graphene Cladding. Appl. Sci. 2021, 11, 10833. https://doi.org/10.3390/ app112210833

Academic Editor:

Antonio Di Bartolomeo

Received: 30 September 2021

Accepted: 11 November 2021

Published: 16 November 2021

Publisher's Note: MDPI stays neutral with regard to jurisdictional claims in published maps and institutional affiliations.

Copyright: (c) 2021 by the authors. Licensee MDPI, Basel, Switzerland. This article is an open access article distributed under the terms and conditions of the Creative Commons Attribution (CC BY) license (https:// creativecommons.org/licenses/by/ $4.0 /)$.

\begin{abstract}
This work presents the role of graphene in improving the performance of a porous GaNbased UV photodetector. The porous GaN-based photodetector, with a mean pore diameter of $35 \mathrm{~nm}$, possessed higher UV sensitivity, about $95 \%$ better compared to that of the as-received (non-porous) photodetector. In addition, it exhibits a lower magnitude of leakage current at dark ambient, about $70.9 \mu \mathrm{A}$, compared to that of the as-received photodetector with $13.7 \mathrm{~mA}$. However, it is also highly resistive in nature due to the corresponding electrochemical process selectively dissolute doped regions. Herein, two types of graphene, derived from CVD and the electrochemical exfoliation (EC) process, were cladded onto the porous GaN region. The formation of a graphene/porous GaN interface, as evident from the decrease in average distance between defects as determined from Raman spectroscopy, infers better charge accumulation and conductance, which significantly improved UV sensing. While the leakage current shows little improvement, the UV sensitivity was greatly enhanced, by about $460 \%$ and $420 \%$ for CVD and EC cladded samples. The slight difference between types of graphene was attributed to the coverage area on porous GaN, where CVD-grown graphene tends to be continuous while EC-graphene relies on aggregation to form films.
\end{abstract}

Keywords: graphene cladding; porous GaN; high sensitivity; UV photodetector

\section{Introduction}

Graphene is best described as a single layer of carbon atoms arranged hexagonally on a flat two-dimensional (2D) plane. It is also known as a zero-gap material with excellent electronic and thermal properties [1-3]. Several known methods such as chemical vapour deposition (CVD), electrochemical exfoliation (EC), Hummers, and so on have gained interest in the scientific community for graphene production [3-11].

Graphene films of lower defects and thickness of monolayers can be grown on copper substrate using the CVD method [10-13]. This method employs vacuum furnace and flow meters, as well as hydrocarbon precursors (e.g., methane) and hydrogen, in order to facilitate the necessary chemical reactions. The aforementioned reactions ascribed to the decomposition of vapour phase hydrocarbons under hot $\left(\sim 1000{ }^{\circ} \mathrm{C}\right)$ and reducing (hydrogen) environment subsequently condensed on the copper surface, which catalyses graphene growth. Given the costly production, other alternatives have been explored as well.

The EC methods operate through the insertion of chemical ions between graphene layers within a graphitic structure. This mechanism is also known as intercalation. Ions of larger size such as $\mathrm{SO}_{4}{ }^{2-}$ would be suitable to be intercalated between graphene layers, 
which leads to structural expansion. Consequently, this weakens the van der Waals forces between graphene layers, making them easier to exfoliate. The exfoliation can be performed using various methods, including microwave, sonication, and so on. With optimization, micron-sized graphene flakes can be obtained [3,14,15].

Gallium nitride $(\mathrm{GaN})$ belonged to the family of group III-nitrides. GaN, a type of semiconductor with a direct bandgap of $3.4 \mathrm{eV}$, is essential in optoelectronics applications such as light-emitting diodes (LEDs) and ultraviolet (UV) photodetectors (PDs) [16-18]. In recent years, nanostructured $\mathrm{GaN}$ has gained popularity in terms of producing more sensitive and efficient devices with higher surface-area-to-volume ratios. One of the known nanostructured $\mathrm{GaNs}$ is porous $\mathrm{GaN}$, which is obtained through UV-assisted photoelectrochemical etching (PEC) of thin-film GaN $[19,20]$. The PEC of GaN can be achieved with or without biasing potential under either acidic or alkaline medium, provided the sample was kept illuminated with a UV light source. Among the options, the presence of biasing potential ensured the uniform etching of GaN. In either acidic or alkaline medium, the etching commenced preferably in the doped region, where the resistance was low. As a result, the post etched region would be highly resistive; however, this feature can be exploited in the fabrication of sensors [21].

Photodetectors (PDs) in general are important components in various optical systems ranging from medical to military applications [22-25]. PDs in general can be categorized into two groups based on their operating mode, i.e., photovoltaic and photoconductive mode [22]. One major difference is the necessity of external bias, where the former is selfoperating, while the latter requires an external bias for better charge transport. It is noteworthy that photovoltaic PDs have great importance in sustainability as they self-operate, with one notable work from Song et al., investigating the performance of MXene/GaN UV PDs [26]. On the other hand, photoconductive PDs are equally important in other sectors.

While a variety of PDs has been produced annually, there is still ongoing research on further improving their performance. Under demanding situations, a good PD should be able to operate with high speed, responses, sensitive, stable, and well-defined bandwidth. These criteria, however, are highly influenced by the surroundings and the sensing material. One particular example is PDs operating at the UV range, where constant illumination from such a wavelength would induce localized heating, potentially affecting the PDs' sensitivity [27]. Conventionally, such PDs are derived from Si-based sensors with photomultiplier tubes, which consisted of complex architecture. With the discovery of wide bandgap semiconductor materials such as $\mathrm{GaN}$, its inherent thermal and chemical stability ensure stable performance $[28,29]$.

Despite the positive aspects of $\mathrm{GaN}$, it faces several challenges in limiting its role as a PD. Due to the limited choice of substrates that matches with the lattice of GaN, defects such as dislocations would be formed during growth. Meanwhile, the intrinsic nature of $\mathrm{GaN}$ requires doping to achieve a reasonable conductivity. With low activation energy, a higher concentration of dopants would be introduced during growth to compensate for such losses. Consequently, this would reduce carrier mobility, thus leading to a slower response [28].

Application of graphene in optoelectronic devices was made possible since it is highly optically transparent $(97.7 \%$ with monolayer) while retaining good electrical conductivity with charge mobility exceeding $100,000 \mathrm{~cm}^{2} \mathrm{~V}^{-1} \mathrm{~s}^{-1}[30,31]$. In recent years, graphene was sought out as the potential material in further enhancing the performance of PDs. The was owed to the unique band structure of graphene, which can be described as the two vertices of inverted triangles meeting at a singular point known as the Dirac Point [32]. The lack of a notable bandgap infers broadband operation, i.e., it is capable of photodetection at a wider range of wavelengths. Next, due to the stable $\mathrm{sp}^{2}$ hybridization of carbon atoms, graphene lacks dangling bonds that enable better charge mobility. In addition, the weak Fermi Level Pining (FLP) effect lowers the potential barrier at the heterojunction, which enables better sensing for PDs [31,33-36]. This also infers that graphene heterojunctions would have rectifying behaviour in general. Examples of graphene heterojunction involving 
semiconductor material such as GaN, GaAs, and $\mathrm{Si}$ to make optical devices would be rectifying in nature [37-41]. Despite the numerous advantages of graphene/semiconductor heterojunction devices, they are susceptible to changes such as defects at the heterojunction. This necessitates studies to be conducted for such heterojunctions.

In this work, a total of four UV photodetectors were fabricated and compared. They were categorized into two groups, with or without graphene cladding. The former consisted of two samples, i.e., as-grown and porous morphology $\mathrm{GaN}$, which acted as control samples. On the other hand, the porous $\mathrm{GaN}$ in the latter group was cladded with graphene grown either using chemical vapour deposition or the electrochemical exfoliation method. The role of this graphene on the performance of a porous GaN-based UV photodetector is compared and evaluated.

\section{Materials and Methods}

This work can be divided into two stages, namely device fabrication and sensing evaluation. The device fabrication stage comprised synthesis of porous $\mathrm{GaN}$ and graphene materials and assembling the UV photodetector. For sensing evaluation, the photodetector will be tested on its current-voltage (IV), sensitivity-transient, and response/recovery time under both dark and UVA illumination.

\subsection{Fabrication of Porous GaN}

In this work, porous $\mathrm{GaN}$ was fabricated using a photo-electrochemical etching (PEC) method. Prior to that, a 2-inch GaN/sapphire wafer was diced into pieces of $12.7 \times 6.35 \mathrm{~mm}^{2}$. The cleaved wafers were sonicated in isopropyl alcohol (IPA) and acetone for $5 \mathrm{~min}$ each to remove surface impurities. Next, they were washed in deionized (DI) water, and finally dried under $\mathrm{N}_{2}$ stream.

A two-electrode electrochemical setup was used to fabricate porous GaN. The GaN wafer served as the anode, while that of $\mathrm{Pt}$ wire was the cathode. Both electrodes were partially immersed in an electrolyte solution, consisting of a mixture of $0.5 \mathrm{M} \mathrm{H}_{2} \mathrm{SO}_{4}$ and $\mathrm{H}_{3} \mathrm{PO}_{4}$. A UV light source was illuminated on the GaN wafer prior to applying $20 \mathrm{~V}$ across the electrodes. Both processes are necessary to initiate the formation of porous morphology on the GaN wafer. The overall process lasted for $30 \mathrm{~min}$. The anodized GaN wafer was subsequently removed and thoroughly washed with DI water in order to remove acidic residues.

\subsection{Synthesis of Graphene}

Both CVD and the electrochemical method were used to synthesize graphene in this work. In the CVD growth, $\mathrm{Cu}$ foil $\left(6.35 \times 6.35 \mathrm{~mm}^{2}\right.$, Strem, $\left.>99.9 \%\right)$ was loaded into a horizontal tube furnace. The furnace temperature subsequently was raised to $800{ }^{\circ} \mathrm{C}$. The $\mathrm{Cu}$ foil was subjected to $\mathrm{H}_{2}$ annealing (flow rate of 150 standard cubic centimetres per minute (sccm)) for 10 min to improve its crystallinity. Next, heptane vapour and $\mathrm{H}_{2}$ gas were channelled into the furnace with (1 standard cubic foot per hour (scfh) and $150 \mathrm{sccm}$, respectively) to initiate graphene growth. The process lasted for $30 \mathrm{~min}$. Finally, the furnace was cooled down naturally, and the graphene/Cu sample was removed.

Electrochemically exfoliated graphene was performed in a two-electrode electrochemical cell, with a graphite rod being the anode, while that of Pt was the cathode. A mixture of $\mathrm{H}_{2} \mathrm{SO}_{4}$ and $\left(\mathrm{NH}_{4}\right)_{2} \mathrm{~S}_{2} \mathrm{O}_{8}$ (both $0.25 \mathrm{M}$ ) would serve as the electrolyte. A total of $2.5 \mathrm{~V}$ was applied across the electrodes to trigger the wetting of the graphite rod. After $5 \mathrm{~min}$, the potential was raised to $5 \mathrm{~V}$, which initiates the exfoliation process. Exfoliated graphene sediments were notably seen within $30 \mathrm{~min}$. Next, the sediments were vacuum filtered and washed several times to remove traces of electrolyte. Finally, the graphene powder was dried and stored in a vial. 


\subsection{Fabrication of UV Photodetectors}

The graphene cladding deposited on porous GaN can be described schematically in Figure 1 and as follows: on CVD grown graphene, a layer of polymethyl methacrylate (PMMA) was spin-coated on the graphene layer and baked at $100{ }^{\circ} \mathrm{C}$ for $2 \mathrm{~min}$ to harden. Next, the underneath $\mathrm{Cu}$ was etched using concentrated $\mathrm{FeCl}_{3}$ solution. The suspended PMMA/graphene was subsequently scooped and washed several times with DI water before being overlaid on porous GaN. Finally, the CG-GaN sample was washed with acetone in order to remove the PMMA layer. A total of four PDs were fabricated and their nomenclature and electrode positions were listed in Table 1.

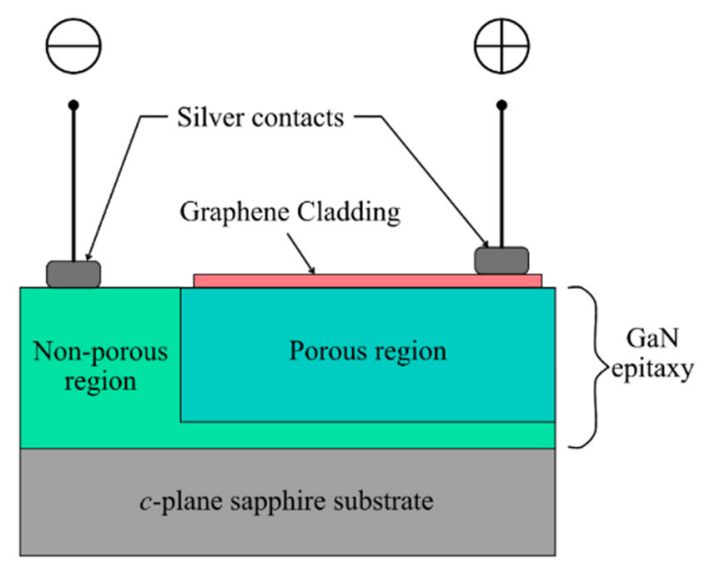

Figure 1. Schematic drawing (not to scale) of the UV photodetector.

Table 1. List of UV photodetectors along with nomenclature in this work.

\begin{tabular}{cc}
\hline Type of Sample/Nomenclature & ${ }^{\mathbf{1}}$ Electrical Contacts Location \\
\hline As-received & Both ends \\
Porous GaN/(Pr-GaN) & Porous (partial), non-porous \\
${ }^{2}$ CVD-graphene-GaN/CG-GaN & Graphene (partial), non-porous \\
${ }^{2}$ EC-graphene-GaN/EC-GaN & Graphene (partial), non-porous \\
\hline
\end{tabular}

${ }^{1}$ Conductive silver paste was used as electrical contacts. ${ }^{2}$ The graphene cladding is located on top of porous GaN only.

For electrochemically exfoliated graphene, $20 \mathrm{mg}$ of EC-graphene powder was dispersed and sonicated in $\mathrm{N}, \mathrm{N}$-dimethylformamide (DMF) for $1 \mathrm{~h}$. Then, the dispersion was left overnight to allow heavier unexfoliated graphite chunks to sediment. The graphene suspension was centrifuged at $4000 \mathrm{rpm}$ for $2 \mathrm{~h}$ subsequently. Next, a few drops of water were added to promote the aggregation of graphene flakes. This was further assisted with the addition of heptane, which trapped the graphene film between heptane/DMF interfaces. The graphene film was drawn onto porous $\mathrm{GaN}$ when the latter descended into the heptane/DMF interface. This method is known as solvent-interface trapping (SIT) [42].

\subsection{UV Photodetector Characterizations}

The morphologies of the UV photodetectors were characterized using a field-emission scanning electron microscope (FEI Nova NanoSEM 450, FEI, Hillsboro, OR, USA). Meanwhile, Raman spectroscopy (Jobin Yvon HR800, HORIBA, Kyoto, Japan) was used to characterize the vibrational modes of graphene. The incident source generated by $\mathrm{Ar}^{+}$laser with a wavelength of $514.5 \mathrm{~nm}$, operating at $20 \mathrm{~mW}$. The UV sensing characteristics, i.e., current-time (I-T) and current-voltage (I-V) of all photodetectors, was performed using a Keithley 4200 source meter, with a UV light source obtained from a UVA black light tube with a peak wavelength of around $355 \mathrm{~nm}$. The optical power density that illuminates the sensor was determined to be $13.6 \mathrm{~mW} / \mathrm{cm}^{2}$. 


\section{Results}

Figure 2 shows the morphologies of as-received $\mathrm{GaN}$, Figure 2a, and porous $\mathrm{GaN}$, Figure $2 \mathrm{~b}$. Prior to PEC etching, the as-received $\mathrm{GaN}$ exhibits a smooth morphology. A porous morphology was obtained upon PEC etching, which is exhibited as dark spots that can be seen in Figure 2b,c. In the latter figure, the magnified view enabled some quantitative analysis, i.e., determining pore diameter to be conducted. Through image processing employing Image J, the statistical data about the pore diameter were obtained and are shown in Figure $2 \mathrm{~d}$. In that figure, the average pore diameter was revealed to be $35 \mathrm{~nm}$.

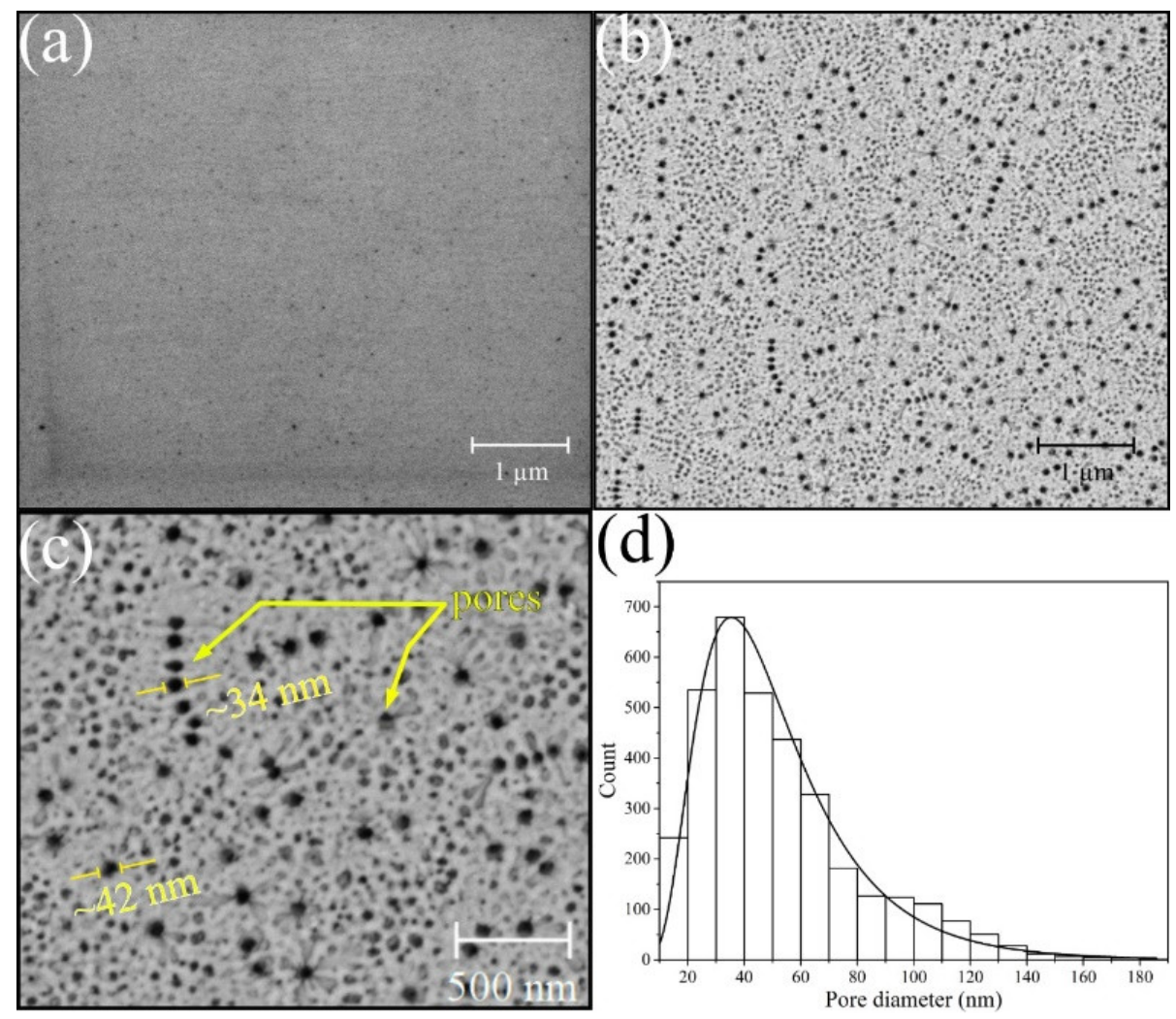

Figure 2. Electron micrographs of (a) as-received $\mathrm{GaN}$, (b) porous $\mathrm{GaN}$, (c) magnified view of porous $\mathrm{GaN}$, and (d) pore diameter distribution of porous $\mathrm{GaN}$.

Figure 3 shows the various types of graphene claddings on porous $\mathrm{GaN}$, and the combination of both also served as the UV sensing layer. In Figure 3a, the area with and without graphene cladding can be seen, with the latter exhibiting a darker contrast. Between CVD-grown and EC-exfoliated graphene, the former shows a continuous film with minor wrinkles (in Figure $3 b$ ) that were ascribed to folding when transferring the film onto porous GaN. The latter, as shown in Figure 3c, was exhibited as micron-sized flakes, which showed discontinuity at various areas, and otherwise overlapped onto one another, which can be seen in the yellow circle in Figure 3d.

Figure 4 shows the compilation of Raman spectra for various samples. In Figure 4a, Raman spectra between the as-received and porous $\mathrm{GaN}$ are displayed. From there, both spectra exhibit identical peak positions at 569 and $737 \mathrm{~cm}^{-1}$, which attribute to the $\mathrm{E}_{2}(\mathrm{HI})$ and $\mathrm{A}_{1}(\mathrm{LO})$ mode of $\mathrm{GaN}$ [43]. It should be noted that the $\mathrm{GaN}$ is $c$-oriented and the Raman configuration adopted was $z(x$, unpolarized $) \bar{z}$; hence, such modes would be allowed. Upon PEC etching, additional modes were revealed. These modes were deemed forbidden under this Raman configuration; however, their existence also infers the successful formation of a porous network, where Raman scattering would take place along the pore walls, which consisted of non-c-oriented facets. The additional modes detected 
were centred around 533 and $561 \mathrm{~cm}^{-1}$, which were ascribed to the $A_{1}(T O)$ and $E_{1}(T O)$ modes of GaN, respectively [43].

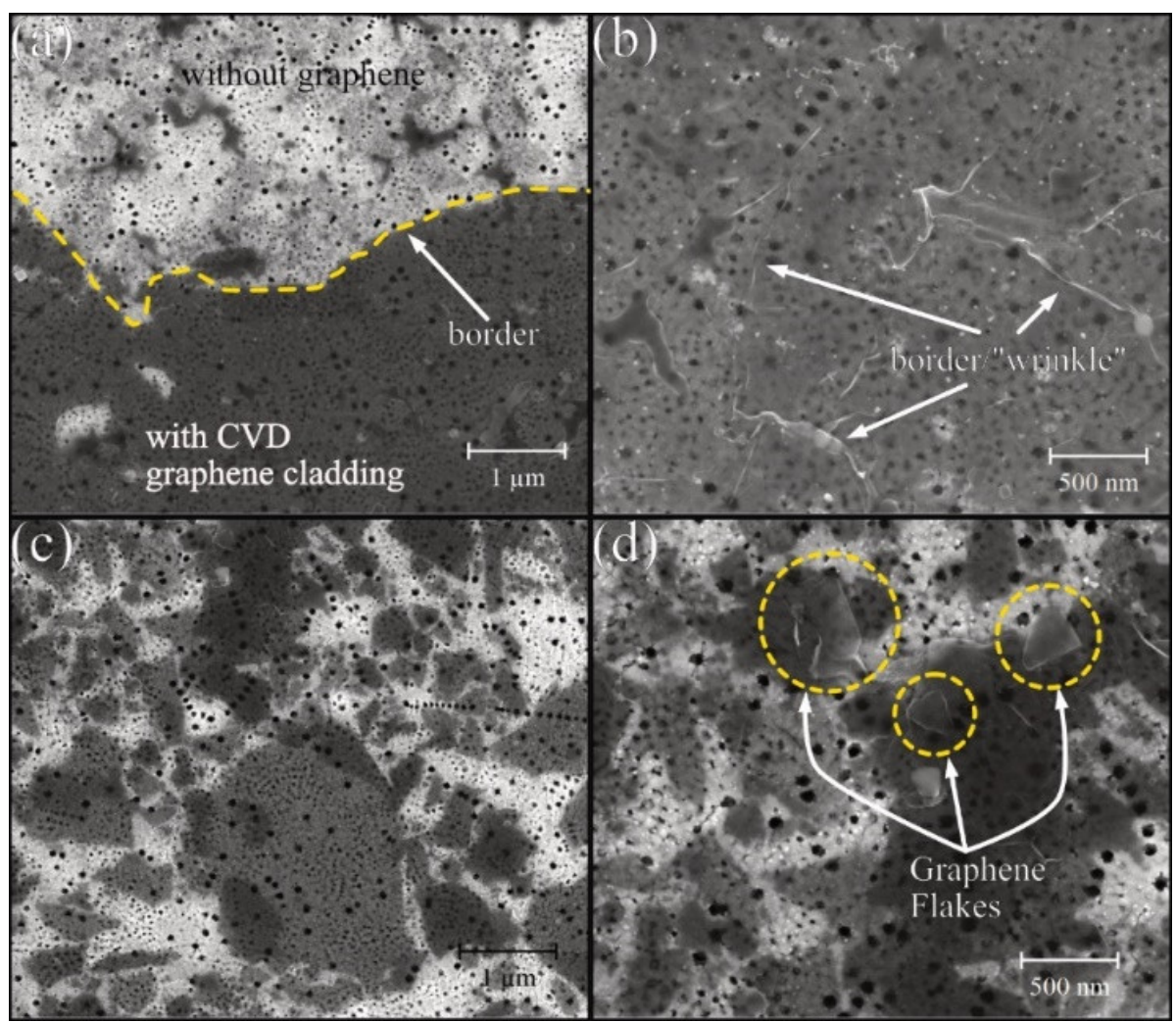

Figure 3. Electron micrographs of various graphene claddings (a) CVD-grown, showing contrast difference between area with and without graphene cladding; (b) CVD-grown, exhibiting continuous film; (c) micron-sized EC-exfoliated graphene flakes; (d) magnified view of EC-exfoliated graphene flakes.
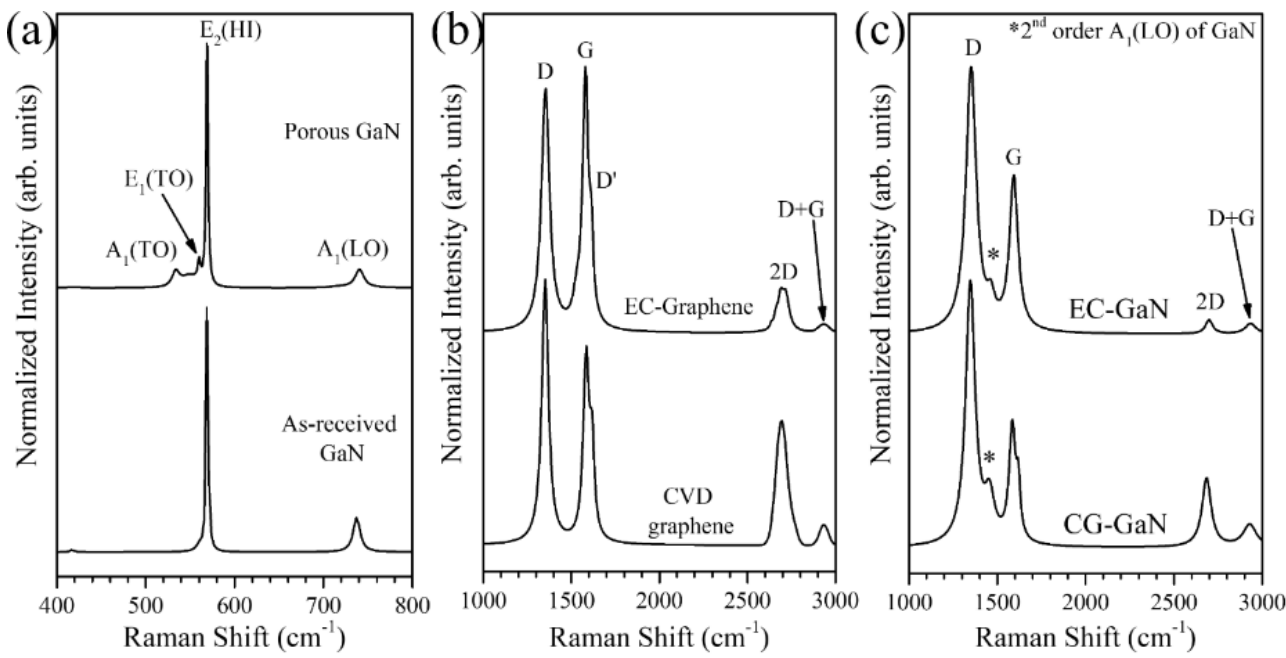

Figure 4. Raman spectra of (a) GaN, (b) graphene, and (c) graphene cladded on porous GaN.

The Raman spectra of CVD-grown and EC-exfoliated graphene are presented in Figure $4 \mathrm{~b}$. Common peaks attributed to graphene can be observed around 1530 and $1589 \mathrm{~cm}^{-1}$, which are assigned to the D and G bands, respectively [44]. On the other hand, Figure 4c shows the Raman spectra of graphene cladding on the porous GaN surface. Compare to Figure $4 \mathrm{~b}$, an additional peak at $\sim 1460 \mathrm{~cm}^{-1}$ attributed to the second-order 
$\mathrm{A}_{1}(\mathrm{LO})$ mode of GaN was spotted [45]. While no significant peak shift occurred in the $\mathrm{D}$ and $G$ peaks relative to that in Figure $4 \mathrm{~b}$, the intensity ratio, $\mathrm{I}_{\mathrm{D}} / \mathrm{I}_{\mathrm{G}}$, changed. Meanwhile, the $2 \mathrm{D}$ bands remained lower than the $\mathrm{G}$ bands for both samples. This feature is ascribed to the presence of multilayer graphene.

Figure 5 shows the current density-voltage $(\mathrm{J}-\mathrm{V})$ curves of all four UV photodetectors taken under dark (Figure 5a) and UV (Figure $5 b$ ) conditions. Rectifying behavior was mostly observed in mostly all photodetectors, with the as-received GaN being less significant. From there, photodetectors consisting of porous morphology clearly exhibited lower current compared to that of as-received GaN. This was ascribed to the mechanism of porous $\mathrm{GaN}$ formation, where doped regions were selectively etched. Consequently, this resulted in a higher resistance, which translates to a significant decrease in current upon biasing.

\section{(a)}

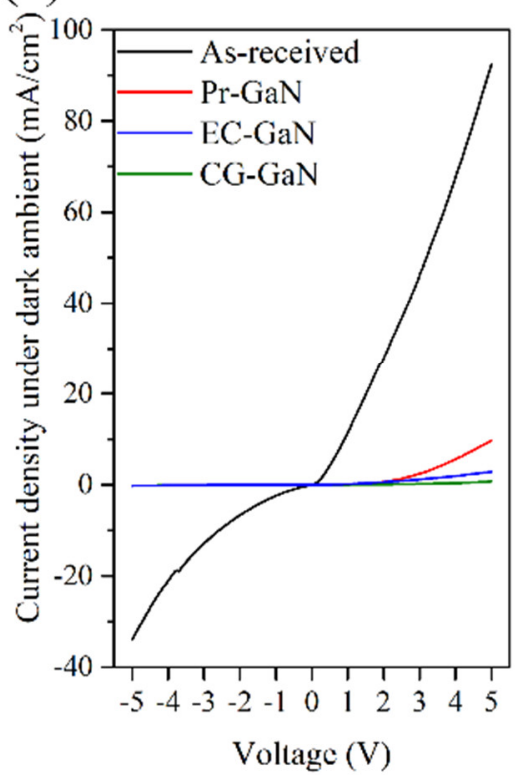

(b)

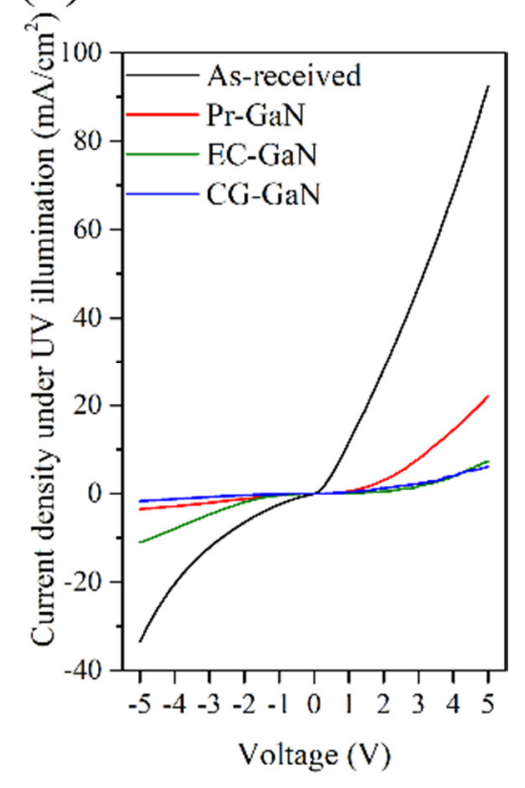

Figure 5. J-V characteristics of UV photodetectors operating under (a) dark and (b) UV ambient.

Figure 6 shows the sensitivity-transient profile of all four UV photodetectors. The sensitivity, $S$, can be evaluated using the following formula:

$$
S=\frac{I_{U V}-I_{\text {dark }}}{I_{\text {dark }}}
$$

where $S$ is sensitivity, $I_{U V}$ is the current measured under UV illumination, and $I_{\text {dark }}$ is the current measured under a dark condition.

The bias voltage was set to $5 \mathrm{~V}$ for all measurements. Among the four photodetectors, CG-GaN gave the highest sensitivity, about 4.6, followed by that of EC-GaN ( 4.2), then Pr-GaN ( 0.95), and finally as-received GaN $(\sim 0.01)$.

Following Equation (1), the responsivity, $R_{I}$, and external quantum efficiency (EQE) of the sensors can be determined using the following formulae [22]:

$$
\begin{gathered}
R_{I}=\frac{I_{p h}}{P} \\
E Q E=\frac{h c}{e \lambda} R_{I}
\end{gathered}
$$


where $I_{p h}$ is the photocurrent, $I_{p h}=I_{U V}-I_{d a r k} ; P$ is the incident light power impinged on the sensor's surface; $h$ is the Planck constant; $c$ is the speed of light; $e$ is the electronic charge; and $\lambda$ is the incident wavelength.

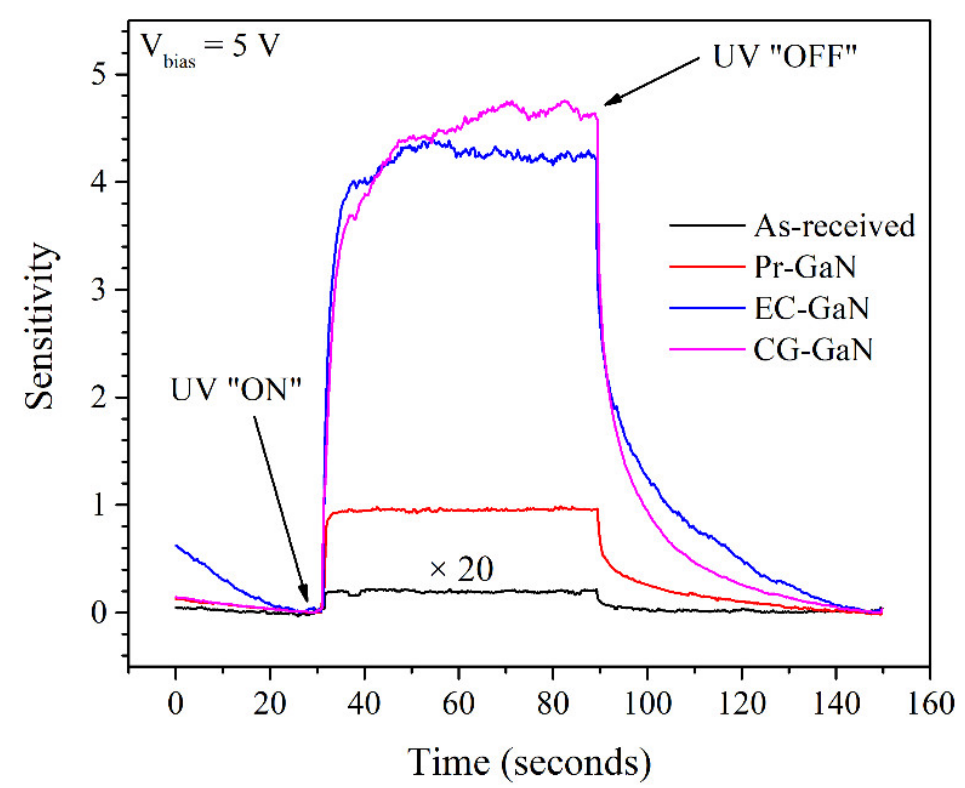

Figure 6. Sensitivity-transient profile of UV photodetectors. The sensitivity for the as-received UV photodetector was multiplied by 20 times for better clarity. The applied biased voltage used in this measurement was set to $5 \mathrm{~V}$.

Since both responsivity and EQE are dependent on the magnitude of photogenerated current, they scaled accordingly to that of sensitivity. The as-received GaN possessed the lowest responsivity of about $0.066 \mathrm{~A} / \mathrm{W}$, with $23 \% \mathrm{EQE}$. Both of these parameters experienced a significant increase when the morphology of $\mathrm{GaN}$ was made porous, with responsivity and EQE of 0.59 and 206\% respectively. By cladding the porous GaN surface with graphene, improved responsivity and EQE was noted; however, when comparing among the types of graphene claddings, their differences were lesser. The highest recorded responsivity and EQE in this work were $1.23 \mathrm{~A} / \mathrm{W}$ and $430 \%$, respectively, when CVDgrown graphene was used as cladding.

From Figure 6, besides the responsivity and EQE, the response and recovery time of each photodetector can also be estimated. The response and recovery time can be defined as the time taken for the sensor sensitivity to rise from 0 to $63.2 \%$ and from 100 to $32.8 \%$, respectively. To summarize the obtained results, they were tabulated in Table 2.

Table 2. Summary of photodetector parameters in this work.

\begin{tabular}{cccccc}
\hline $\begin{array}{c}\text { Type of Sample/ } \\
\text { Nomenclature }\end{array}$ & Sensitivity & $\begin{array}{c}\text { Response } \\
\text { Time (s) }\end{array}$ & $\begin{array}{c}\text { Recovery } \\
\text { Time (s) }\end{array}$ & $\begin{array}{c}\text { Responsivity } \\
\text { (A/W) }\end{array}$ & EQE (\%) \\
\hline $\begin{array}{c}\text { As-received } \\
\text { Porous GaN/ } \\
\text { (Pr-GaN) }\end{array}$ & 0.01 & 3.3 & 1.8 & 0.066 & 23 \\
$\begin{array}{c}\text { EC-graphene- } \\
\text { GaN/EC-GaN }\end{array}$ & 0.95 & 0.9 & 7.8 & 0.59 & 206 \\
$\begin{array}{c}\text { CVD-graphene- } \\
\text { GaN/CG-GaN }\end{array}$ & 4.2 & 2.6 & 4.8 & 1.11 & 390 \\
\hline
\end{tabular}

The as-received GaN exhibited the greatest response time, about $3.3 \mathrm{~s}$, compared to those with porous morphology. This is ascribed to the separation of photogenerated carriers occurred at the surface, where the higher surface area in Pr-GaN would be advantageous 
in producing more photocurrent, providing a more responsive response. The addition of graphene cladding raises the response time, with EC- and CG-GaN having response times of 1.2 and 2.6 s, respectively. Meanwhile, the recovery times for Pr-, CG-, and EC-GaN remained higher than their response time, varying from 4.8 to $8.7 \mathrm{~s}$.

\section{Discussions}

The formation of porous morphology on GaN through PEC can be ascribed to the following chemical reaction [21]:

$$
2 \mathrm{GaN}+6 \mathrm{~h}^{+} \rightarrow 2 \mathrm{Ga}^{3+}+\mathrm{N}_{2}
$$

where GaN underwent dissolution in an acidic medium in the presence of photogenerated carriers due to UV light illumination. This electrochemical process occurred preferentially towards the doped region, hence leading to the formation of a porous network. The average pore diameter as determined from the histogram was $35 \mathrm{~nm}$, and with densely packed pores distributed throughout the porous GaN surface, this led to higher surface area that promoted greater sensing compared to that of the as-received GaN.

$\mathrm{GaN}$ in general possessed a hexagonal wurtzite structure, with a space group of $\mathrm{C}_{6}^{4}{ }_{6 \mathrm{~V}}$. Under group theory, a total of eight sets of phonon modes were predicted measured from $\Gamma=0$ in the Brillouin zone. These modes were categorized in this manner: $2 \mathrm{E}_{2}$, $2 A_{1}, 2 E_{1}$, and $2 B_{1}$. From there, one of the $A_{1}$ and $E_{1}$ modes was Raman active, while all of the $B_{1}$ modes were silent [46]. In Figure $4 a$, the as-received GaN was comprised of two notable peaks that were ascribed to $E_{2}(\mathrm{HI})$ and $\mathrm{A}_{1}(\mathrm{LO})$. When correlated to the Raman configuration of $z(x$, unpolarized $) \bar{z}$, it is discernible that the as-received $\mathrm{GaN}$ was $c$-oriented. Due to the dissolution of GaN through PEC etching, other facets have been exposed, and as light scattering occurred along the pore channels, modes that were deemed forbidden under the aforementioned Raman configuration such as $E_{1}(T O)$ and $A_{1}(T O)$ would be shown.

The differences among Raman spectra, seen in Figure $4 b, c$, have provided some insights into the interaction between graphene and the porous GaN surface. Prior to that, the characteristics of the graphene film need to be studied. Through studying the Raman spectra in Figure $4 b$, it can be seen that CVD-grown graphene possessed higher $I_{D} / I_{G}$ than that of EC-exfoliated graphene. This is largely attributed to the growth mechanism of CVD graphene employing heptane as a precursor. For typical CVD graphene, hydrocarbon precursors, such as methane, underwent stepwise decomposition under hydrogen ambient, subsequently condensed on the copper surface. Due to the poor solubility of carbon towards copper, additionally being the mono carbon atom, the condensed carbon atoms would have more room for crystallization, which resulted in larger grains. In contrast to the use of heptane (heptane contained seven carbon atoms), the decomposition of a longer hydrocarbon chain would be less efficient, hence resulting in smaller crystallites being formed.

The $\mathrm{I}_{\mathrm{D}} / \mathrm{I}_{\mathrm{G}}$ ratio can be further used to determine the average crystallite size $\left(\mathrm{L}_{\mathrm{a}}\right)$ and average distance between defects $\left(\mathrm{L}_{\mathrm{D}}\right)$ by employing the Tuinstra-Koenig relation [47]:

$$
\begin{gathered}
\left(\mathrm{L}_{\mathrm{D}}\right)^{2}\left(\mathrm{~nm}^{2}\right)=\left(1.8 \times 10^{-9}\right) \times \lambda^{4} \times\left(\frac{\mathrm{I}_{\mathrm{D}}}{\mathrm{I}_{\mathrm{G}}}\right)^{-1} \\
\mathrm{~L}_{\mathrm{a}}(\mathrm{nm})=\left(2.4 \times 10^{-10}\right) \times \lambda^{4} \times\left(\frac{\mathrm{I}_{\mathrm{D}}}{\mathrm{I}_{\mathrm{G}}}\right)^{-1}
\end{gathered}
$$

where $\lambda$ is the wavelength of the incident Raman laser, which is $514.5 \mathrm{~nm}$ in this work.

The $\mathrm{L}_{\mathrm{a}}$ for EC- and CVD graphene were 17.5 and $11.6 \mathrm{~nm}$, while those of $\mathrm{L}_{\mathrm{D}}$ were 11.5 and $9.3 \mathrm{~nm}$. The comparable $L_{a}$ to $L_{D}$ infer that defects would be located at the edges of graphene crystallites. This leaves the in-plane domains having available $\pi$-bonds that would interact with the porous GaN surface. 
Figure 4c shows the Raman spectra of graphene cladding on the porous $\mathrm{GaN}$ surface. Compare to Figure $4 \mathrm{~b}$, an additional peak $\sim 1460 \mathrm{~cm}^{-1}$ was attributed to the second-order $\mathrm{A}_{1}(\mathrm{LO})$ mode of $\mathrm{GaN}$ [45]. It was noted that the $\mathrm{I}_{\mathrm{D}} / \mathrm{I}_{\mathrm{G}}$ ratios for both samples have increased relative to that without GaN. By using the Tuinstra-Koenig relation, the $\mathrm{L}_{\mathrm{D}}$ for both EC- and CVD-graphene cladding can be determined, i.e., 8.5 and $7.4 \mathrm{~nm}$, respectively. The decrease of $L_{D}$ was expected as it signified the presence of interfacial interaction between graphene and porous GaN. Graphene in general acted as a topology insulator, where charge conduction performed better within the basal plane. This leaves the outer $\pi$ clouds uninterrupted. As the porous $\mathrm{GaN}$ surface was resistive due to losses of carriers through PEC etching, having the $\pi$ clouds from the graphene layers resulted in interfacial interaction. This effect was exhibited when electrical characterizations were performed.

The analysis of Figure 5a leads to some interesting findings. The current magnitude at the $5 \mathrm{~V}$ reference point was shown to decrease according to the photodetectors of asreceived GaN, Pr-GaN, EC-GaN, and CG-GaN. While the decrease was attributed to the resistive nature of porous $\mathrm{GaN}$, the graphene claddings in general were electrically conductive, which suggests higher current compare to that of Pr-GaN. However, the contrary occurred. This implies that graphene cladding served other roles as well. One plausible role is the formation of a space-charge region at the graphene/GaN interface, which impedes the flow of charge unless sufficient energy was supplied. When comparing EC- to CG-GaN, the latter shows even lower current. This can be correlated to the distribution of graphene on the porous surface of GaN. CVD-grown graphene possessed continuous domains that provide good surface coverage compared to that of EC-GaN, which in turn led to more interfacial interactions.

Another interesting feature is the negative bias region, where the current magnitude was significantly lower for photodetectors with porous morphology. This feature can be attributed to leakage current, where lower magnitude is desirable for photodetectors. From there, the as-received $\mathrm{GaN}$ would have a higher leakage current compared to others, i.e., $-13.7 \mathrm{~mA}$ at $-5 \mathrm{~V}$. This was attributed to the carriers in as-received $\mathrm{GaN}$ being higher compared to the other samples. Upon undergoing PEC, the leakage current significantly decreased, being $-70.9 \mu \mathrm{A}$ or $0.52 \%$ relative to that of as-received GaN. The inclusion of graphene claddings seemed to contribute lesser changes towards leakage current, with ECand CVD-graphene cladding contributing to -83.5 and $-63.6 \mu \mathrm{A}$, respectively.

In Figure 5, some changes in current magnitude were observed for photodetectors upon UV light illumination. Interestingly, in the negative bias region, the leakage current seemed to increase for photodetectors with porous morphology, with the exception of CG-GaN having the least leakage current among the others. This substantiates the role of graphene cladding with good coverage to impede the flow of charges in the negative bias region. Although the photodetector exhibited responses towards UV light illumination in the form of an increase in current magnitude, current-transient responses would be more suitable for providing a clearer picture.

The variations of sensitivity for various photodetectors as shown in Figure 6 remained intriguing. By comparing as-received and Pr-GaN, it was expected the latter would have higher sensitivity compared to the former due to the significant increase in surface area. Interestingly, upon cladding the porous $\mathrm{GaN}$ surface with graphene, the sensitivity was further enhanced. Given the morphological aspects of graphene, the enhanced sensitivity was less likely contributed by an increase in surface area. Based on the earlier results from Figures 4 and 5, interfacial interaction between porous $\mathrm{GaN}$ and graphene has led to the formation of a space-charge region. This region is not only better at impeding charges flow in the reverse bias condition but also provides a shorter path length for charge transportation upon UV illumination. This would translate into higher sensitivity for such sensors. Meanwhile, when comparing both CG- and EC-GaN photodetectors, their difference in sensitivity was less notable. However, CG-GaN would retain a higher sensitivity compared to EC-GaN, which can be attributed to the distribution of graphene cladding. CVD-grown graphene is regarded as a continuous film, hence providing better 
cladding compared to that of electrochemical exfoliation, which requires control over surface tension in order to aggregate graphene flakes that could still provide small voids upon successful SIT deposition.

The response time for the photodetectors was reduced when made into porous morphology, additionally cladded with graphene. The rise can be ascribed to the additional time required to overcome the space-charge region between graphene and the porous GaN surface. Meanwhile, the slight difference between EC- and CG-GaN was likely due to the coverage of graphene on porous GaN. Since CVD-grown graphene provides better coverage, this also inferred more space-charge regions between interfaces. Despite the improved response time, the recovery time behaved otherwise. The increase in recovery time was attributed to the resistive nature of the photodetectors. While being responsive towards photogenerated carriers, the photodetectors have become more resistive, while the presence of space-charge regions in graphene-cladded photodetectors has to be taken into account. This inferred more time required to dissipate the remaining photogenerated carriers when sensing the ambient changes from UV light illumination to dark.

Based on the obtained experiment results, the sensing mechanism of the UV sensor in this work was proposed and translated figuratively in Figure 7 . Since the UV sensors require an external bias to operate, they can be regarded as operating under a photoconductive mode. For as-received GaN, as shown in Figure 7a, two metal contacts consisting of conductive silver paint were applied on both ends of the sample. The exposed surface would serve as an active layer where photogenerated carriers would be induced upon UV light illumination. Since GaN is capable of absorbing UV light owing to its wide bandgap, photogenerated carriers would preferably form near the surface. This also implies the number of photogenerated carriers would be lesser compared to the other sensors in this work; hence, lower sensing would be expected. The J-V characteristics of this sensor were almost linear, with little signs of rectifying behaviour with large leakage current. This was likely due to the symmetrical placement of the silver contacts on the GaN surface; hence, charge flow was possible in either direction. In addition, silver has a lower metal work function; hence, the barrier between silver/GaN was less notable, enabling charge flow with lesser resistance.

(a)

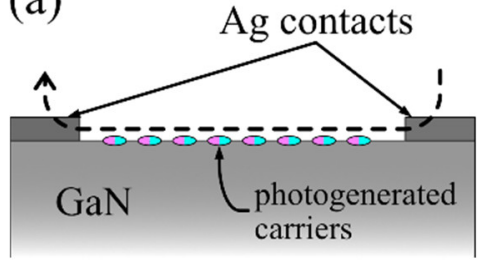

(b)
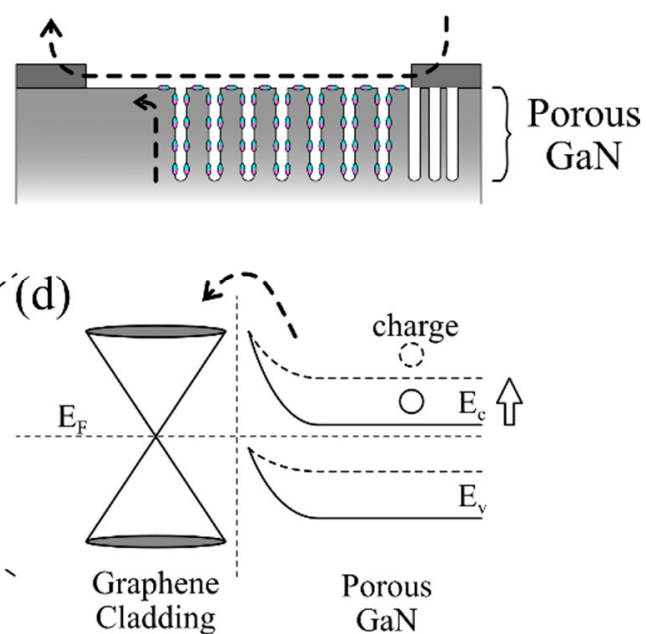

Figure 7. Suggested sensing mechanism of the UV photodetectors based on (a) as-received GaN, (b) Pr-GaN, (c) both EC- and CG-GaN, and (d) band diagram illustrating the conduction of charges at the graphene/porous GaN heterojunction.

For Pr-GaN, the surface of GaN has been etched to reveal a porous morphology. This has significantly increased its surface area, which leads to higher absorption of UV light along the pore channels. Consequently, the additional photogenerated carriers would lead 
to higher sensitivity. The J-V characteristics for this sensor exhibit discernible rectifying behaviour. This was likely due to the surface passivation of porous $\mathrm{GaN}$, where a rather thin layer of oxide formed on the surface of GaN. The passivated layer would induce a potential barrier between the porous $\mathrm{GaN}$ interface, trapping photogenerated carriers until sufficient energy was supplied to properly conduct them.

Upon introducing graphene cladding to porous $\mathrm{GaN}$, an additional mechanism was included. This involved the electrical polarization at the graphene-porous $\mathrm{GaN}$ interface. The $\mathrm{sp}^{2}$ hybridization of carbon in graphene leads to unbound charges at the $\pi$-orbital, or $\pi$-cloud, that permit interaction with various compounds. In this work, a built-in electric field was induced at the graphene/porous $\mathrm{GaN}$ interface, which led to more notable rectifying behaviour. Since the placement of metals contacts was not symmetrical, i.e., one on the graphene surface and the other on the GaN (non-porous) surface, charge conduction became polarization-dependent, which led to the suppression of current flow in the reverse bias configuration. Although the graphene/porous GaN heterojunction possessed a builtin electric field, the weak Fermi-level pinning has eased charge migration from porous $\mathrm{GaN}$ to graphene, which leads to enhanced sensing performance.

\section{Conclusions}

Graphene has been synthesized using two methods, namely CVD and EC. The graphene materials were cladded onto the surface of porous $\mathrm{GaN}$ ( $\mathrm{Pr}-\mathrm{GaN})$, where the overall product would be a UV-photodetector, and subsequently, their performances were evaluated. Between the two types of graphene claddings, there were some notable differences. Morphologically, the CVD method could be used to grow a continuous 2D graphene sheet, while micron-sized graphene flakes were obtained using the EC method. Despite both types of graphene having almost similar crystallite size $\mathrm{L}_{\mathrm{a}}$ and comparable average distance between defects $\mathrm{L}_{\mathrm{D}}$. Although Pr-GaN had a higher exposed surface area for UV sensing, its performance was significantly improved with the integration of graphene, i.e., graphene/Pr-GaN heterojunction. Through such integration, the $\mathrm{L}_{\mathrm{D}}$ was slightly reduced. However, in terms of performance, CG-GaN showed the highest response, followed by EC$\mathrm{GaN}$, with sensitivities of 4.6 and 4.2 , respectively. It should be noted that there were lesser differences between the aforementioned in this manner. On the responsivity and EQE, it was found that the as-received GaN possessed values of $0.066 \mathrm{~A} / \mathrm{W}$ and $23 \%$, respectively, while CG-GaN showed $1.23 \mathrm{~A} / \mathrm{W}$ and $430 \%$. The presence of the potential barrier was deduced to be present for Pr-GaN in the form of surface passivation, hence resulting in rectifying behaviours in the $\mathrm{J}-\mathrm{V}$ profile. The inclusion of graphene claddings induced a built-in electric field at the heterojunction that further suppressed reverse leakage current, while serving as a current collector to further enhance the sensitivity of the photodetector.

Author Contributions: Conceptualization, K.P.B., A.R., N.M.A. and Z.H.; methodology, K.P.B. N.M.A., A.R. and F.K.Y.; software, F.K.M. and K.P.B.; validation, K.P.B., A.R. and N.M.A.; formal analysis, F.K.M. and K.P.B.; investigation, F.K.M. and K.P.B.; resources, K.P.B., A.R. and N.M.A.; data curation, F.K.M. and K.P.B.; writing — original draft preparation, F.K.M. and K.P.B.; writing-review and editing, K.P.B., A.R., N.M.A. and F.K.Y.; visualization, F.K.M. and K.P.B.; supervision, K.P.B., A.R. and N.M.A.; project administration, K.P.B., F.K.Y. and Z.H.; funding acquisition, K.P.B. All authors have read and agreed to the published version of the manuscript.

Funding: This research was funded by the short-term grant of Universiti Sains Malaysia (USM), grant number 304/PFIZIK/6315307.

Acknowledgments: The work is financially supported by the short-term grant of Universiti Sains Malaysia (USM), titled "Template-Assisted Growth of Group III-Nitrides Nanomaterials and Their Integration in Sensing Applications", with grant no.: 304/PFIZIK/6315307. The first author would like to express her gratitude and thankfulness for IPS in sponsoring her work through the graduate research assistance (GRA) scheme. In addition, all supports from USM, IPS, and INOR are gratefully acknowledged.

Conflicts of Interest: The authors declare no conflict of interest. 


\section{References}

1. Dong, L.-X.; Chen, Q. Properties, synthesis, and characterization of graphene. Front. Mater. Sci. China 2010, 4, 45-51. [CrossRef]

2. Tao, Q.; Wu, R.; Li, Q.; Kong, L.; Chen, Y.; Jiang, J.; Lu, Z.; Li, B.; Li, W.; Li, Z.; et al. Reconfigurable electronics by disassembling and reassembling van der Waals heterostructures. Nat. Commun. 2021, 12, 1825. [CrossRef] [PubMed]

3. Kim, D.S.; Jeong, J.M.; Park, H.J.; Kim, Y.K.; Lee, K.G.; Choi, B.G. Highly Concentrated, Conductive, Defect-free Graphene Ink for Screen-Printed Sensor Application. Nanomicro Lett. 2021, 13, 87. [CrossRef]

4. Loryuenyong, V.; Totepvimarn, K.; Eimburanapravat, P.; Boonchompoo, W.; Buasri, A. Preparation and Characterization of Reduced Graphene Oxide Sheets via Water-Based Exfoliation and Reduction Methods. Adv. Mater. Sci. Eng. 2013, $2013,1-5$. [CrossRef]

5. Ye, S.; Ullah, K.; Zhu, L.; Ali, A.; Jang, W.K.; Oh, W.-C. CVD growth of large-area graphene over Cu foil by atmospheric pressure and its application in $\mathrm{H}_{2}$ evolution. Solid State Sci. 2015, 46, 84-88. [CrossRef]

6. Yu, P.; Lowe, S.E.; Simon, G.P.; Zhong, Y.L. Electrochemical exfoliation of graphite and production of functional graphene. Curr. Opin. Colloid Interface Sci. 2015, 20, 329-338. [CrossRef]

7. Liu, H.; Liu, Y. Controlled Chemical Synthesis in CVD Graphene. Phys. Sci. Rev. 2017, 2, 104-145. [CrossRef]

8. Chen, Z.; Qi, Y.; Chen, X.; Zhang, Y.; Liu, Z. Direct CVD Growth of Graphene on Traditional Glass: Methods and Mechanisms. Adv. Mater 2019, 31, 1803639. [CrossRef]

9. Le Ba, T.; Mahian, O.; Wongwises, S.; Szilágyi, I.M. Review on the recent progress in the preparation and stability of graphenebased nanofluids. J. Therm. Anal. Calorim. 2020, 142, 1145-1172. [CrossRef]

10. Liu, Y.; Feng, H.; Luo, F. Quantitative analysis of the defects in CVD grown graphene by plasmon-enhanced Raman scattering. Carbon 2020, 161, 153-161. [CrossRef]

11. Guermoune, A.; Chari, T.; Popescu, F.; Sabri, S.S.; Guillemette, J.; Skulason, H.S.; Szkopek, T.; Siaj, M. Chemical vapor deposition synthesis of graphene on copper with methanol, ethanol, and propanol precursors. Carbon 2011, 49, 4204-4210. [CrossRef]

12. Komissarov, I.V.; Kovalchuk, N.G.; Kolesov, E.A.; Tivanov, M.S.; Korolik, O.V.; Mazanik, A.V.; Shaman, Y.P.; Basaev, A.S.; Labunov, V.A.; Prischepa, S.L.; et al. Micro Raman investigation of graphene synthesized by atmospheric pressure CVD on copper foil from decane. Phys. Procedia 2015, 72, 450-454. [CrossRef]

13. Al-Hazmi, F.; Beall, G.W.; Al-Ghamdi, A.; Alshahrie, A.; Shokr, F.; Mahmoud, W.E. Raman and ellipsometry spectroscopic analysis of graphene films grown directly on Si substrate via CVD technique for estimating the graphene atomic planes number. J. Mol. Struct. 2016, 1118, 275-278. [CrossRef]

14. Yang, S.; Bruller, S.; Wu, Z.S.; Liu, Z.; Parvez, K.; Dong, R.; Richard, F.; Samori, P.; Feng, X.; Mullen, K. Organic Radical-Assisted Electrochemical Exfoliation for the Scalable Production of High-Quality Graphene. J. Am. Chem. Soc. 2015, 137, 13927-13932. [CrossRef]

15. Parvez, K.; Yang, S.; Feng, X.; Müllen, K. Exfoliation of graphene via wet chemical routes. Synth. Met. 2015, 210, 123-132. [CrossRef]

16. Yang, J.; Wei, T.; Hu, Q.; Huo, Z.; Sun, B.; Duan, R.; Wang, J. Green light emitting diode grown on thick strain-reduced GaN template. Mater. Sci. Semicond. Process. 2015, 29, 357-361. [CrossRef]

17. Park, J.-S.; Han, J.; Seong, T.-Y. Improving the output power of GaN-based light-emitting diode using Ag particles embedded within a $\mathrm{SiO}_{2}$ current blocking layer. Superlattices Microstruct. 2015, 83, 361-366. [CrossRef]

18. Beh, K.P.; Yam, F.K.; Tan, L.K.; Ng, S.W.; Chin, C.W.; Hassan, Z. Photoelectrochemical Fabrication of Porous GaN and Their Applications in Ultraviolet and Ammonia Sensing. Jpn. J. Appl. Phys. 2013, 52, 08JK03. [CrossRef]

19. Yam, F.K.; Hassan, Z.; Chuah, L.S.; Ali, Y.P. Investigation of structural and optical properties of nanoporous GaN film. Appl. Surf. Sci. 2007, 253, 7429-7434. [CrossRef]

20. Xiao, H.; Cui, J.; Cao, D.; Gao, Q.; Liu, J.; Ma, J. Self-standing nanoporous GaN membranes fabricated by UV-assisted electrochemical anodization. Mater. Lett. 2015, 145, 304-307. [CrossRef]

21. Vajpeyi, A.P.; Chua, S.J.; Tripathy, S.; Fitzgerald, E.A. Effect of carrier density on the surface morphology and optical properties of nanoporous GaN prepared by UV assisted electrochemical etching. Appl. Phys. Lett. 2007, 91, 083110. [CrossRef]

22. Long, M.; Wang, P.; Fang, H.; Hu, W. Progress, Challenges, and Opportunities for 2D Material Based Photodetectors. Adv. Funct. Mater. 2018, 29, 1803807. [CrossRef]

23. Li, Z.; Liu, X.; Zuo, C.; Yang, W.; Fang, X. Supersaturation-Controlled Growth of Monolithically Integrated Lead-Free Halide Perovskite Single-Crystalline Thin Film for High-Sensitivity Photodetectors. Adv. Mater. 2021, 33, 2103010. [CrossRef]

24. Chen, Y.; Su, L.; Jiang, M.; Fang, X. Switch type PANI/ZnO core-shell microwire heterojunction for UV photodetection. J. Mater. Sci. Technol. 2022, 105, 259-265. [CrossRef]

25. Crisci, T.; Moretti, L.; Casalino, M. Theoretical Investigation of Responsivity/NEP Trade-off in NIR Graphene/Semiconductor Schottky Photodetectors Operating at Room Temperature. Appl. Sci. 2021, 11, 3398. [CrossRef]

26. Song, W.; Chen, J.; Li, Z.; Fang, X. Self-Powered MXene/GaN van der Waals Heterojunction Ultraviolet Photodiodes with Superhigh Efficiency and Stable Current Outputs. Adv. Mater. 2021, 33, 2101059. [CrossRef]

27. Casalino, M. Silicon Meets Graphene for a New Family of Near-Infrared Schottky Photodetectors. Appl. Sci. 2019, 9, 3677. [CrossRef]

28. Aggarwal, N.; Gupta, G. Enlightening gallium nitride-based UV photodetectors. J. Mater. Chem. C 2020, 8, 12348-12354. [CrossRef] 
29. Gao, N.; Fang, X. Synthesis and Development of Graphene-Inorganic Semiconductor Nanocomposites. Chem. Rev. 2015, 115, 8294-8343. [CrossRef] [PubMed]

30. Bonaccorso, F.; Sun, Z.; Hasan, T.; Ferrari, A.C. Graphene photonics and optoelectronics. Nat. Photonics 2010, 4, 611-622. [CrossRef]

31. Yao, J.D.; Yang, G.W. All-2D architectures toward advanced electronic and optoelectronic devices. Nano Today 2021, $36,101026$. [CrossRef]

32. Yao, J.D.; Zheng, Z.Q.; Yang, G.W. Production of large-area 2D materials for high-performance photodetectors by pulsed-laser deposition. Prog. Mater. Sci. 2019, 106, 100573. [CrossRef]

33. An, Y.; Behnam, A.; Pop, E.; Ural, A. Metal-semiconductor-metal photodetectors based on graphene/p-type silicon Schottky junctions. Appl. Phys. Lett. 2013, 102, 013110. [CrossRef]

34. Liu, C.H.; Chang, Y.C.; Norris, T.B.; Zhong, Z. Graphene photodetectors with ultra-broadband and high responsivity at room temperature. Nat. Nanotechnol. 2014, 9, 273-278. [CrossRef]

35. Garg, R.; Dutta, N.K.; Choudhury, N.R. Work Function Engineering of Graphene. Nanomaterials 2014, 4, 267-300. [CrossRef]

36. Pant, R.; Singh, D.K.; Chowdhury, A.M.; Roul, B.; Nanda, K.K.; Krupanidhi, S.B. Next-generation self-powered and ultrafast photodetectors based on III-nitride hybrid structures. APL Mater. 2020, 8, 020907. [CrossRef]

37. Yoo, D.; Lee, K.; Tchoe, Y.; Guha, P.; Ali, A.; Saroj, R.K.; Lee, S.; Islam, A.; Kim, M.; Yi, G.C. Dimension- and position-controlled growth of GaN microstructure arrays on graphene films for flexible device applications. Sci. Rep. 2021, 11, 17524. [CrossRef]

38. Skachkov, D.; Liu, S.-L.; Wang, Y.; Zhang, X.-G.; Cheng, H.-P. First-principles theory for Schottky barrier physics. Phys. Rev. B 2021, 104, 045429. [CrossRef]

39. Tao, Z.; Zhou, D.; Yin, H.; Cai, B.; Huo, T.; Ma, J.; Di, Z.; Hu, N.; Yang, Z.; Su, Y. Graphene/GaAs heterojunction for highly sensitive, self-powered Visible/NIR photodetectors. Mater. Sci. Semicond. Process. 2020, 111, 104989. [CrossRef]

40. Fidan, M.; Ünverdi, Ö.; Çelebi, C. Junction area dependent performance of graphene/silicon based self-powered Schottky photodiodes. Sens. Actuators A Phys. 2021, 331, 112829. [CrossRef]

41. Zulkifli, N.A.A.; Park, K.; Min, J.-W.; Ooi, B.S.; Zakaria, R.; Kim, J.; Tan, C.L. A highly sensitive, large area, and self-powered UV photodetector based on coalesced gallium nitride nanorods/graphene/silicon (111) heterostructure. Appl. Phys. Lett. 2020, 117, 191103. [CrossRef]

42. Woltornist, S.J.; Oyer, A.J.; Carrillo, J.-M.Y.; Dobrynin, A.V.; Adamson, D.H. Conductive thin films of pristine graphene by solvent interface trapping. ACS Nano 2013, 7, 7062-7066. [CrossRef] [PubMed]

43. Kuball, M. Raman spectroscopy of GaN, AlGaN and AlN for process and growth monitoring/control. Surf. Interface Anal. 2001, 31, 987-999. [CrossRef]

44. Ferrari, A.C.; Meyer, J.C.; Scardaci, V.; Casiraghi, C.; Lazzeri, M.; Mauri, F.; Piscanec, S.; Jiang, D.; Novoselov, K.S.; Roth, S.; et al. Raman spectrum of graphene and graphene layers. Phys. Rev. Lett. 2006, 97, 187401. [CrossRef]

45. Davydov, V.Y.; Kitaev, Y.E.; Goncharuk, I.; Smirnov, A.; Graul, J.; Semchinova, O.; Uffmann, D.; Smirnov, M.; Mirgorodsky, A.; Evarestov, R. Phonon dispersion and Raman scattering in hexagonal GaN and AlN. Phys. Rev. B 1998, 58, 12899-12907. [CrossRef]

46. Kuball, M.; Pomeroy, J.W.; Rajasingam, S.; Sarua, A.; Uren, M.J.; Martin, T.; Lell, A.; Härle, V. High spatial resolution micro-Raman temperature measurements of nitride devices (FETs and light emitters). Phys. Status Solidi (a) 2005, 202, 824-831. [CrossRef]

47. Beams, R.; Gustavo Cancado, L.; Novotny, L. Raman characterization of defects and dopants in graphene. J. Phys. Condens Matter 2015, 27, 083002. [CrossRef] 